\title{
Practice-based evidence from a clinical cohort that received pediatric constraint- induced movement therapy
}

\author{
Stephanie C. DeLuca ${ }^{\mathrm{a}, \mathrm{b}, \mathrm{c}, \mathrm{e}, *}$, Mary Rebekah Trucks ${ }^{\mathrm{a}}$, Dorian A. Wallace ${ }^{\mathrm{a}}$ and Sharon L. Ramey,b,c,d \\ ${ }^{a}$ Virginia Tech Carilion Research Institute, Roanoke, VA, USA \\ ${ }^{\mathrm{b}}$ Department of Pediatrics, Virginia Tech Carilion School of Medicine, Roanoke, VA, USA \\ ${ }^{\mathrm{c}}$ Department of Psychology, Virginia Tech, Blacksburg, VA, USA \\ ${ }^{\mathrm{d}}$ Department of Psychiatry and Behavioral Medicine, Virginia Tech Carilion School of Medicine, Roanoke, VA, \\ USA \\ ${ }^{\mathrm{e}}$ Department of Rehabilitation and Wellness, Jefferson College of Health Sciences, Roanoke, VA, USA
}

Accepted 26 November 2016

\begin{abstract}
.
PURPOSE: Constraint-Induced Movement Therapy (CIMT) is now designated a highly efficacious treatment for children with cerebral palsy, based on rigorous clinical trials. Yet virtually no evidence confirms that these moderate to large size effects can be replicated in clinical practice for a more heterogeneous clinical population. Thus there is a need to collect and report treatment outcome data based on actual clinical practice as a critical next step for implementation.

METHODS: This study presents results from a prospective study conducted on a clinical cohort of 88 children, 18 months to 12 years old ( $\mathrm{M}=55$ months, $\mathrm{SD}=5$ months), who received high-intensity CIMT known as ACQUIREc. The children varied in severity and etiology of their hemiparesis and a subset was diagnosed with asymmetric quadriparesis.

RESULTS: Pre- to post-CIMT assessments confirmed highly significant and clinically meaningful changes based on both parental report (Pediatric Motor Activity Log, $p<0.0001$ ) and standardized measures (The Assisting Hand Assessment, $p=$ $0.04)$.

CONCLUSIONS: Clinical practice of high-intensity CIMT (120 hours in 4 weeks) with full-time casting of the less-impaired upper extremity produced benefits of comparable magnitude to those from rigorous randomized controlled trials (RCTs). Therapists were highly trained and actively monitored. Children across a wide range of etiologies and severity levels realized positive outcomes.
\end{abstract}

Keywords: Practice-based evidence, constraint-induced movement therapy, hemiparesis, quadriparesis, cerebral palsy

\section{Introduction}

Pediatric Constraint-Induced Movement Therapy (CIMT) repeatedly has produced significant functional gains in children with hemiparesis [1-4]. For children

\footnotetext{
${ }^{*}$ Corresponding author: Stephanie C. DeLuca, Virginia Tech Carilion Research Institute, 2 Riverside Circle, Roanoke, VA 24016 , USA. Tel.: +1 540526 2098; E-mail: stephdeluca@vt.edu.
}

with cerebral palsy (CP), CIMT has been tested in at least 14 randomized controlled trials (RCTs) and has been the subject of many independent systematic reviews [5-8] concluding that there is strong evidence supporting its efficacy [5]. Accordingly, CIMT has been designated as the most highly recommended form of intervention for children with hemiparetic $\mathrm{CP}$ [5]. Over the past decade, numerous professional workshops and training courses have been offered on CIMT; and many clinics and practicing therapists 
have endeavored to provide some form of CIMT to their patient populations. Many families and therapists have embraced its core ideas of high-intensity therapy and use of constraint of the less-impaired upper extremity generating a myriad of CIMT protocol variations [4-9]-most typically labeled as modified or adapted CIMT. The compelling question is whether clinical practice can produce the same type and magnitude of benefits as those reported in rigorous RCTs?

Implementation science is the relatively new branch of scientific investigation that explores how to move evidence-based practices into routine clinical care. Often there is a need to determine the extent to which the originally tested treatment protocol is followed in clinical practice and the extent to which variations in key components of the treatment protocols contribute to differential outcomes in patient populations. For the adult CIMT intervention, developed for patients secondary to stroke, Reiss et al. [10] proposed that variations in protocols could be classified as signature forms if they are of much higher intensity and specify more directly how to use reinforcement and shaping methods and as modified forms when of lesser intensity and therapy specificity. For the field of pediatric CIMT, Ramey, Coker-Bolt, and DeLuca [3,11] proposed a similar designation and identified five core treatment components: 1) constraint of the unimpaired or less impaired arm and hand; 2) relatively high dosage therapy consisting of many treatment hours each day and multiple days per week; 3) use of operant conditioning techniques (reinforcement, shaping through successive approximations) and massed practice to promote motor learning; 4) providing therapy in a natural environment; and 5) a post-CIMT transition plan. Ramey et al. [3,11] further differentiated signature pediatric CIMT provides all five core elements at very high levels (including dosage levels) from the modified and adapted forms that often include only the two distinctive features that Eliasson et al. proposed in 2014 [12] namely "(1) restraint of the well-functioning upper limb (irrespective of device/type) and (2) intensive structured training (irrespective of type)." Similar to Ramey et al., Eliasson et al. also recognized both signature and modified forms of pediatric CIMT. For both theory and clinical practice, resolving the unanswered questions about the importance of dosage and the type of constraint used will be important. Several groups have proposed a dosage of at least 3 hours of therapy each day for 5 days a week for a minimum of 2 weeks. Of note, two comparative effectiveness RCTs been funded by NIH that are addressing these important variations in signature and modified forms of pediatric CIMT (footnote 1) - trials that fulfill the high priority research needs identified independently by Ramey et al. and by Eliasson et al. in their reviews.

After two decades of research on pediatric CIMT, there are two noteworthy contributions to the pediatric rehabilitation community that extend beyond the topic of efficacy per se. First is the demonstration that a precisely defined treatment protocol is not at odds with individualization of the treatment goals and the use of child- and family-friendly best practices (e.g., building on the child's expressed interests, incorporating play and activities of daily living into the treatment protocol, scheduling treatment at times convenient for the child and family). Pediatric occupational therapists and physical therapists have viewed individualized therapy as paramount to the success of rehabilitation (although this has never been tested). Indeed, when conventional or widely used forms of rehabilitation therapies have failed to demonstrate efficacy under scientific scrutiny, clinicians have remained skeptical about these findings because of this issue regarding individualization. A second and closely related contribution concerns the prominent need to manualize therapy protocols in order to ensure that distinctive components, such as the therapy content and dosage, are operationalized and thus adhered to when an efficacious treatment makes the transition into clinical care. Although most forms of pediatric CIMT that have been tested in rigorous clinical trials do not have treatment manuals available, one form does - namely, ACQUIREc [15] that was used in the first RCT of pediatric CIMT which started in 1998 and was first published in 2004 by Taub et al., and again in the first multi-site RCT [1,16-18]. In this article, we provide data about a clinical practice that sought to replicate ACQUIREc in its clinical services. The establishment of the clinic required rigorous training for all therapists and creating a treatment schedule that allowed them to treat children for 6-hour daily sessions, for at least 5 days a week, for 4 consecutive weeks and required therapists to maintain systematic documentation of daily treatment activities and the child's behavioral responses.

There are, of course, many practical and administrative concerns when clinics or individual therapists seek to set up processes for the delivery of CIMT. CIMT is delivered in a highly intensive burst, in contrast to traditional rehabilitation that is distributed in single sessions, usually 1 hour in duration (and some- 
times less) across an extended time. Can clinics adapt therapeutic practices for delivering services in intensive bursts? Further, this signature form of pediatric CIMT known as ACQUIREc requires providing treatment in the child's home or a homelike setting, such as apartment for families who travel to receive this specialized treatment. To the extent that clinics seeking to deliver evidence-based practices also are willing to provide careful, objective documentation of their treatment delivery and to assess children, before and after receiving therapy, then clinical practices can advance knowledge about real-world variations and expected outcomes. This approach to generating practice-based evidence [13] (PBE) complements findings from rigorous RCTs and serves to provide practical evidence needed to expand the implementation of new therapies. PBE calls for using clinical cohorts to study large heterogeneous clinical populations as new treatments are developed, proven efficacious, and then have their results disseminated. In 2014 Ammerman et al. [14] stated that "The most threatening public health challenges today are chronic and complex and require joint effort from academic researchers in partnership with clinical and public health practitioners to identify and implement sustainable solutions that work in the real world. Practice-based research offers researchers and practitioners an underutilized way forward, an opportunity to work together to design and test feasible, evidence-based programs to address our greatest challenges."

This study examines a signature CIMT protocol used at 2 clinical sites. Both of these sites used a PBE model and systematically collected evidence, with parent permission and an IRB-approved data collection protocol, obtaining assessment data appropriate for the child's age, pre- and post-CIMT. In addition, therapists were instructed in how to document daily CIMT activities with written treatment logs and supplemental evidence from videotaping treatment sessions with each child for the purposes of ensuring high levels of fidelity to the implementation of this signature form of CIMT. The clinical population was more heterogeneous than those traditionally targeted in CIMT studies providing a first look at how one CIMT protocol might fare when disseminated into a more diverse $\mathrm{CP}$ population.

\section{Methods}

\subsection{Research design}

The design is a prospective clinical cohort study of children served in one of two university-based centers where ACQUIREc, a signature form of pediatric CIMT $[3,4,11,16]$, was offered. Both centers were a fee-for-service clinic and were created and directed by original developers of ACQUIREc (DeLuca and Ramey). A total of seven different therapists were trained in ACQUIREc following the administrative manual protocol. Data collection and study enrollment protocols were approved by both universities' institutional review boards. All families voluntarily consented prospectively to the collection and use of data. Families were informed and agreed to have each child complete a battery of assessments immediately prior to and after CIMT (within 3 days before and after treatment) that would be used for research purposes. Data about each child's progress during therapy also were collected by the therapist and parent provided ratings about their children's use of the more-impaired upper extremity in everyday activities. Families self-referred their children for services after learning about the clinical facility, most often through contact with families who had previously sought treatment and from reading the published scientific literature. (Note: Neither clinic has ever advertised or solicited to recruit children; further, the clinics were designed to be self-sustaining through fees, but not to generate profit.)

\subsection{Inclusion criteria}

Children were evaluated for medical stability and were required to have documented asymmetric motor function between their upper extremities, but were not excluded for bilateral motor impairment or for cognitive involvement. Medical records were reviewed for specific diagnoses, levels of impairment, and the documentation of cognitive impairment. Levels of impairment were defined by treating therapists. Cognitive impairment was defined as present if the medical records documented such a diagnosis.

The clinics had no age limits and served children between seven months and 21 years of age, delivering more than 400 full ACQUIREc treatment bursts of a month. The sample included in this report includes only those children who were receiving CIMT for the first time and who were between 18 months and 12 years of age. The lower end of this range was chosen based on age requirement for the Assisting Hand Assessment (AHA) (described below) as an outcome measure; the upper age was chosen to focus on children prior to the onset of adolescence. 


\subsection{Intervention}

The ACQUIREc signature form of pediatric CIMT $[12,16]$ was delivered to all children. Constraint of the non-involved upper extremity was accomplished by the construction of a full arm cast that each child wore continuously during the first 15 to 19 treatment days of the total 20 or 21 treatment days. We used focused rigidity casting, a lightweight fiberglass product that allows for varying levels of rigidity to be placed within a final cast. The rigidity for these children was focused at the elbow to keep it in $90^{\circ}$ flexion. The cast itself started just below the shoulder and encompassed the arm and hand through the fingertips with the thumb slightly abducted from the fingers which were in a neutral position.

Therapy itself consisted of a 6 hour treatment session on every weekday, although approximately half of the children received an additional treatment day on one Saturday over the four weeks of treatment. This Saturday session provided an opportunity for some families to have both parents present to observe a full day of treatment and how they could participate in helping their child to practice some of the new skills learned during CIMT. We varied the total number of bilateral treatment days (when the cast was removed) based on clinical judgment about each child and discussions with each family, particularly in relationship to treatment goals the families and children had and the extent to which additional therapist training for bilateral activities was needed. The range of the number of bilateral days was between 3 and 5 treatment days in the last week.

All activities during therapy utilized operant conditioning that involved tasks being partitioned into subdivisions of movements and skills which were reinforced via praise, opportunity to engage in a favorite activity, and/or small tangible rewards (e.g., a favorite snack) for success at a desired level. As proficiency of the movement or skill reached a $70-80 \%$ consistency level, the therapist increased the skill level needed to earn reinforcement. Movements and skills were thus progressively shaped using successive approximations towards the targeted behavior. All 6 hours of daily treatment involved active play-based therapy or activities of daily living with operant conditioning occurring for a wide range of age-appropriate play and selfhelp tasks. For example, both a 24 month old and an 11 year old might work on feeding skills everyday with their involved arm and hand, but an example of a play item for a 24 month old might be using the hemiparetic arm and hand to activate a cause-and-effect light-up toy, whereas a play item for the 11 year old might be a board game or a sports-related skill.

\subsection{Therapist training and treatment fidelity}

Training of therapists occurred for a minimum of one month and often lasted for three months, full-time. Intensive interaction occurred with senior therapists and treatment protocol developers prior to a therapist being allowed to deliver CIMT. The first few children treated by a new therapist were actively supervised, in person, with fidelity checks and feedback occurring to adhere to the ACQUIREc treatment protocol [15-20]. Therapists were required to be highly knowledgeable about the manualized protocol and in all cases worked closely with senior therapists in applying the principles of shaping conjointly at first, followed by a period of time when their therapeutic efforts were documented via videotape and subsequently checked for fidelity of implementation by protocol developers. Ongoing efforts to maintain high levels of fidelity included periodic visits by senior therapists and daily discussions about treatment events including challenges and successes with individual children.

\subsection{Response to constraint in the clinic-based practice}

The cast was removed weekly to allow for active range of motion of the stronger arm and hand and to monitor for potential problems such as skin breakdown or irritation. Skin prickly and minor irritation that involved no break in the skin occurred for about $40 \%$ of the children. In all of these cases, and even when there was no skin irritation, children were allowed to bathe and wash and dry the casted arm and hand. Also, each child practiced a few bilateral skills prior to the cast being reapplied within about 60 minutes. About $10 \%$ greater levels of skin irritation occurred such as the presence of a blister or a skin pinch that led to a break in the skin. We shared this information with the parents and we then altered the cast to either increase padding or remove a pressure point prior to its reapplication. One child had noted swelling in the hand when the cast was removed during the third week of treatment. This case was considered significant because the arm and hand had been normal during the first two weekly checks. Accordingly, we left the cast off for several days, while continuing the full 6 hour treatment sessions each day, and we consulted the child's physician. The swelling did resolve, but to be safe, we chose not to replace the cast during the final treatment week. 


\subsection{Unanticipated 'clinical' events}

The most notable unanticipated event that occurred during treatment was the occurrence of seizures. Seizure activity appeared for two children who had no prior medical seizure history reported. A supervisory therapist noticed seizure activity in one child and referred the family for diagnosis, and in the second undiagnosed child a seizure occurred outside of treatment hours during night-time hours. This child was also referred to a neurologist for seizure diagnosis and management. Two other children who had previously been diagnosed with seizure activity were documented to have a seizure incident during the 4 week treatment period, but these occurred outside treatment hours and did not appear linked to CIMT intervention in any detectable way.

One child developed cold and flu symptoms during the first two days of treatment. Treatment was suspended and delayed for several days until the child felt better. Five children developed cold-like symptoms during the 4 week treatment course that were documented to alter the planned treatment course with 3 children ending a treatment day 15 to 30 minutes early and 2 other children having the treatment activities adjusted they seemed fatigued because of their cold symptoms and thus each had somewhat reduced ability to participate for a few of the treatment days.

\subsection{Assessments}

Three measures of treatment outcome are presented in this article: The Pediatric Motor Activity Log (PMAL), the Emerging Behaviors Scale (EBS), and the Assisting Hand Assessment (AHA). All assessments were completed within three days prior to and three days after CIMT. The assessment battery was delivered by clinical personnel. Because CIMT was the only intervention delivered by this clinic, assessors could not be masked to the type of intervention. However, active supervision of assessment protocols and their scoring sought to maintain high consistency and adherence to each assessment's administration.

The PMAL is a parental report about the child's arm use within their environment. The PMAL is a standardized tool in which parents rate their children's abilities using a 6-point Likert scale on Quality of Movement $(0=$ no use; $5=$ age-typical/normal use $)$ and Frequency of Use ( $0=$ never; $5=$ very frequently) for each of 22 arm and hand activities (e.g., holding a cup/bottle, waving bye, pushing a button to acti- vate a toy, throwing a ball, putting an arm through a sleeve of clothing). Wallen et al. in 2008 [19] reported PMAL psychometrics on a data-transformed version of the PMAL; however, the scale is presented in the current paper as developed by the original authors of the tool $[1,15,16]$.

The Emerging Behaviors Scale (EBS) is a summary score derived from the multiple data collection techniques that counts the number of discrete skills or movement patterns that a child demonstrates in at least two different settings or on two different assessment protocols. The EBS consists of a set of possible 31 discrete UE activities that include movement patterns and functional abilities (e.g. wrist extension, finger isolation, finger feeding, pushing buttons). For the movement or skill to be counted as being present, the child must display this in two or more situations. For example, the behavior could be observed on a video recording of a therapy play session and then independently shown again when the child successfully passes an item that is included in a standardized assessment [13].

The AHA is a measure of the child's use of the weaker arm and hand during bimanual activities and has been psychometrically evaluated for children between 18 months and 12 years of age [17]. The AHA scores are transformed into logit interval level scores for data analysis which have been developed secondary to Rasch analyses. AHA results are only presented for the sample of children diagnosed with unilateral impairment because the authors of the AHA have not reported the validity of the measure for children with bilateral but asymmetric impairment and do not recommend its use in children other than those with unilateral impairment. The AHA scoring for this sample was completed by an AHA certified assessor who used the 5.0 AHA version [21]. The assessor reviewed AHA assessments on an ongoing basis. Further, to the extent possible, the assessor was not informed about whether the AHA session took place prior to or after the child received CIMT.

\subsection{Data analysis}

Planned data analyses included paired samples ttest to look at pre- to post-treatment changes for each outcome measure. Paired samples testing was chosen because it accounts statistically for the known relationship between pre- and post-treatment samples. Outcome measures were based on clinical relevance. There were no defined comparison groups because all children received the same treatment. Specifically, the 
Table 1

Sample demographic characteristics

\begin{tabular}{|c|c|}
\hline \multicolumn{2}{|c|}{ Demographic characteristics $\mathrm{N}=88$} \\
\hline Age & $\begin{array}{c}\mu=54.6 \\
\text { Range } 19-144 \text { months }\end{array}$ \\
\hline \multicolumn{2}{|l|}{ Presentation } \\
\hline Hemiparetic & $77(88 \%)$ \\
\hline Quadriparetic & $10(11 \%)$ \\
\hline \multicolumn{2}{|l|}{ Gender } \\
\hline Males & $41(47 \%)$ \\
\hline Females & $47(53 \%)$ \\
\hline \multicolumn{2}{|c|}{ Cognitive involvement } \\
\hline Yes & $23(26 \%)$ \\
\hline No & $65(74 \%)$ \\
\hline \multicolumn{2}{|l|}{ Side of involvement } \\
\hline Right & $53(60 \%)$ \\
\hline Left & $35(40 \%)$ \\
\hline \multicolumn{2}{|l|}{ Diagnosis } \\
\hline Cerebral palsy & $54(61 \%)$ \\
\hline Stroke & $26(30 \%)$ \\
\hline TBI & $7(8 \%)$ \\
\hline Tumor resection & $1(1 \%)$ \\
\hline \multicolumn{2}{|c|}{ Manual Abilities Classification System } \\
\hline II & $15(17 \%)$ \\
\hline III & $24(27 \%)$ \\
\hline IV & $13(15 \%)$ \\
\hline $\mathrm{V}$ & $3(3 \%)$ \\
\hline *Unassigned & $33(38 \%)$ \\
\hline \multicolumn{2}{|c|}{ Gross Motor Functional Classification System [23] } \\
\hline I & $41(47 \%)$ \\
\hline II & $16(18 \%)$ \\
\hline III & $5(6 \%)$ \\
\hline IV & $1(1 \%)$ \\
\hline $\mathrm{V}$ & $2(2 \%)$ \\
\hline *Unassigned & $23(26 \%)$ \\
\hline
\end{tabular}

* Represents children unable to be prospectively evaluated for classification level.

clinic's interest was to learn more about the degree to which this signature model CIMT could be implemented in a clinic and produce evidence about effectiveness - ideally, at levels comparable to the efficacy data generated via RCTs. Correlations between child age and change scores were computed, and analysis of variance (ANOVA) testing was completed to test for differential responses by demographic and clinical variables of interest.

\section{Results}

\subsection{Sample}

The clinical cohort's diagnostic and demographic variables are presented in Table 1 for 88 children.

The mean PMAL quality of movement rating pretreatment was 1.44 (s.d. $=1.01$ ) with a post-treatment mean of 2.94 (s.d. $=0.94)$ with a resulting $\mathrm{t}=13.40$, $p<0.0001$. The mean PMAL frequency of use rating pre-treatment was 1.10 (s.d. $=0.78$ ) with a posttreatment mean of 3.05 (s.d. $=0.96$ ); $\mathrm{t}=16.14, p<$ 0.0001. The EBS pretreatment mean was 9.97 (s.d. $=$ 5.78 ) with a post-treatment mean 20.73 (s.d. = 5.98); $\mathrm{t}=17.81, p<0.0001$. The AHA mean pretreatment logit score was $37.50(7.05)$ and the post-treatment mean logit was 55.25 (13.07); $\mathrm{t}=3.57, p=0.04$. Figure 1 shows the pre- to post-treatment AHA raw scores.

Age did not have a significant relationship with amount of change on any outcome measure. There also were no significant outcome differences for children between children who received 21 days versus 20 days of CIMT. None of the demographic variables or diagnostic scores showed a significant effect on CIMT outcomes; that is, children of diverse abilities appeared to show comparable levels of pre- and post-CIMT changes - all statistically significant. However, three child characteristics did relate significantly to treatment responsiveness: the child's Manual Abilities Classification System level (MACS) [22], whether the child was diagnosed with hemiparesis (unilateral) versus quadriparesis (bilateral with asymmetry), and whether the child had clinically documented cognitive impairment.

The PMAL indicated a significant difference between the amount of change parents rated on the Frequency of Use Scale of the PMAL by MACS level with an $\mathrm{f}=3.54, p=0.02$. Treatment changes by MACS levels on the PMAL are shown in Fig. 2. Univariate analyses indicate that there were significant pretreatment differences on the PMAL, Quality of Movement Scale based on MACS level, $\mathrm{f}=4.04, \mathrm{p}=0.01$. Follow up analyses and Fig. 2 demonstrate that parents report positive responses to increased changes in the frequency that children are using their arms and hands at all MACS levels but that children at MACS levels I and $\mathrm{V}$ are significantly different than other classification levels. Parents of children with a MACS level of 2 reported the highest amount change in the frequency with which their children used their arm and hands. Parents of children at MACS level V did not report significant changes on the PMAL Quality of Movement Scale. MACS level was not a statistically significant predictor on other measures, although this may be due to the small number of children at some levels. For example, on the EBS the mean number of new behaviors for MACS level II $=9.21$, for level III $=11.26$, for level IV $=9.36$, and for level $\mathrm{V}=13.50$. 


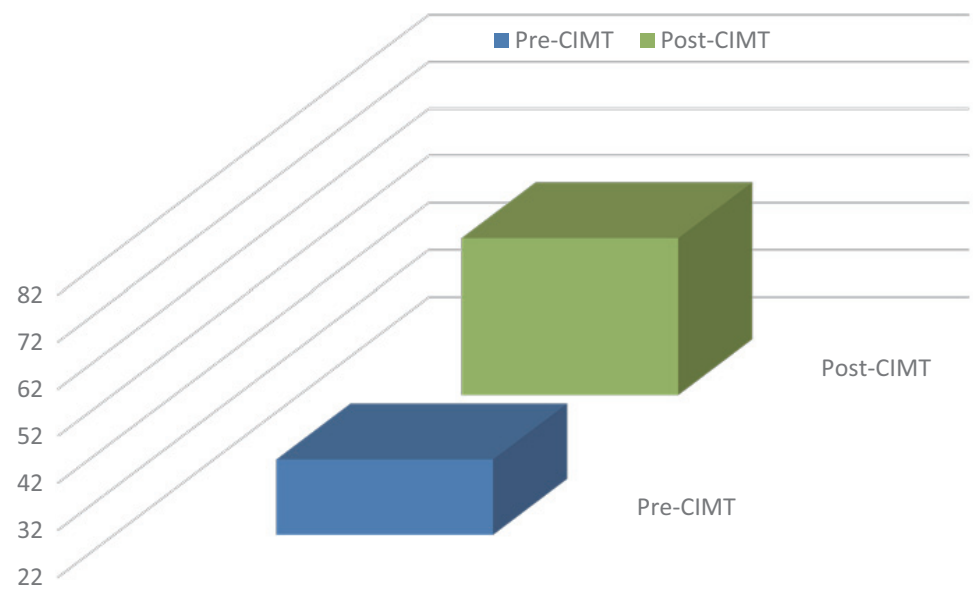

Fig. 1. AHA raw scores pre- to post-CIMT.

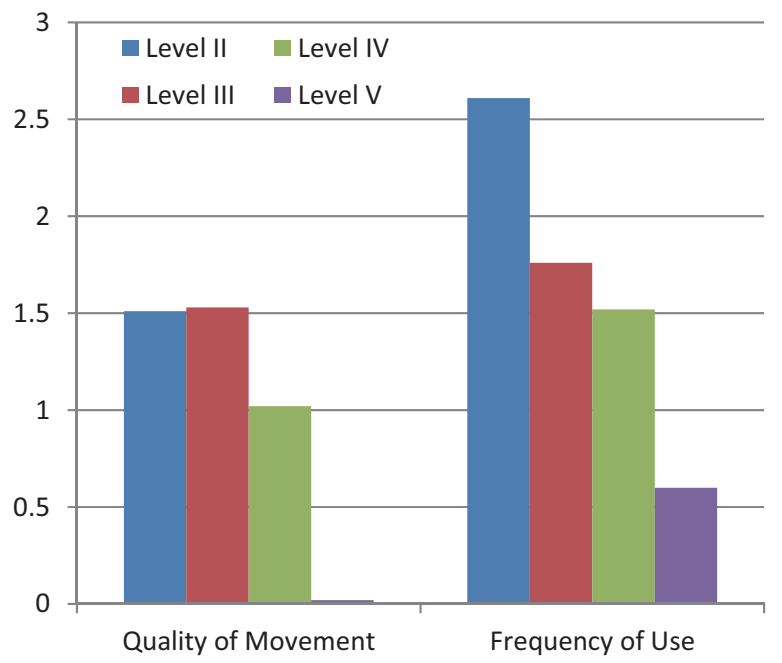

Fig. 2. Change amounts in the pediatric motor activity log by Manual Abilities Classification System.

\section{Discussion}

These findings demonstrate that a signature form of a pediatric CIMT protocol can be successfully implemented in a clinical setting where a heterogeneous sample of children received CIMT to improve their upper extremity function in uni- and bilateral activities. Unlike children in the RCTs, $11 \%$ presented with asymmetric quadriparesis, $26 \%$ had documented cognitive impairments, and children ranged across four of the five MACS classification levels, and across all GMFCS levels. The responsiveness to CIMT in this highly diverse population was positive and statistically significant for all outcomes. In Fig. 3, we compare the percentage of change from baseline from this clini- cal cohort on the parental ratings and on the Emerging Behaviors Scale to results from previous RCTs. The results are highly similar, although the magnitude is somewhat reduced in the more heterogeneous clinical sample. This difference could be due to a number of factors. The clinical sample of children has children with greater impairment levels than previous RCT samples - most notably, the inclusion of children with quadriparesis or bilateral impairment. In addition, the sensitivity of the outcome measures may vary, to unknown degrees, across all functioning levels; this makes it difficult to have a common metric or set of clinical outcome expectations that apply in a similar way across impairment levels and etiological groups. Further, we used measures developed for children with hemiparesis, so applying these to children with quadriparesis may have some limitations or may have missed other important changes that were not captured by these assessments.

There are only a handful of CIMT studies within the literature that report functional classification levels and even fewer that consider functional classifications as a predictor of change $[25,26]$. In this sample, MACS level was a predictor of treatment response in one outcome measure, the PMAL. Parents of children with higher MACS levels reported the greatest amounts of change in both how well and how often their children used their weaker arm and hand, but parents of children with the lowest levels of function (MACS level 5) reported significant gains in how often their children used their arm and hand even though they did not necessarily report high changes in how well their children used their weaker arm and hand. This likely indicates a greater willingness in these children to make 


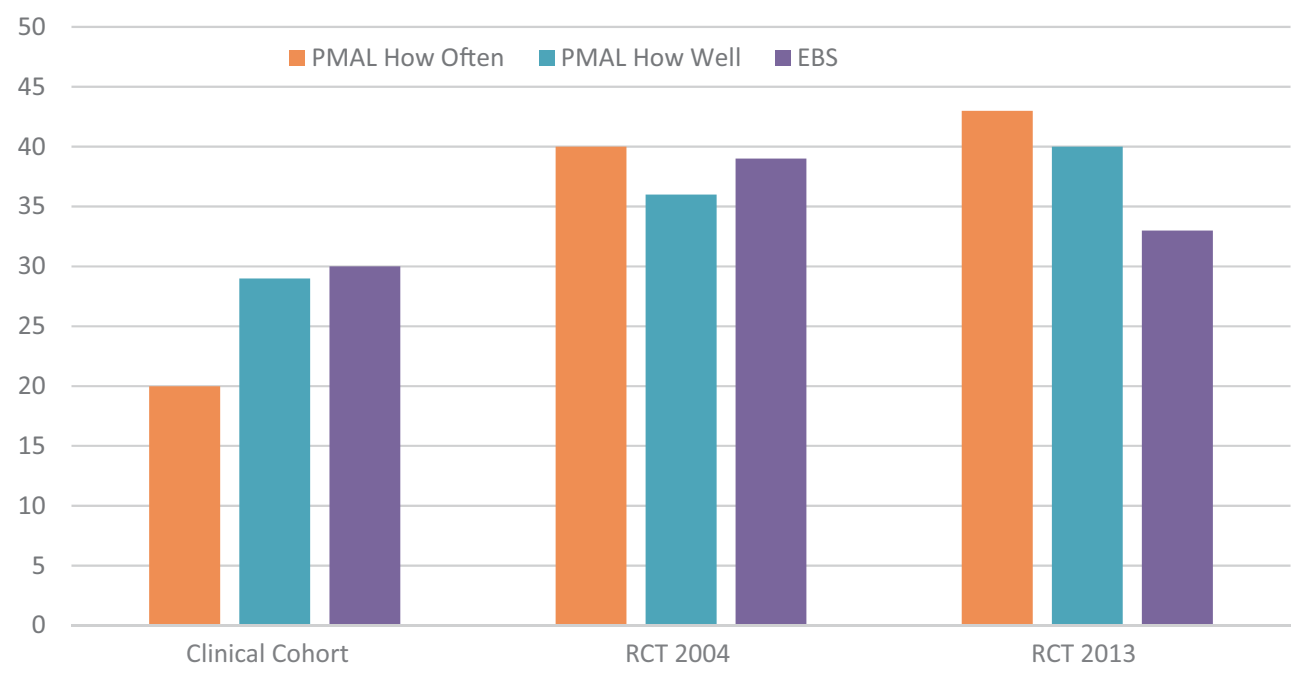

Fig. 3. Percentage change amounts across trials.

attempts to use their weaker arm despite overall functional status. This willingness to use the arm and hand could lead to more therapeutic opportunities, including the possibility that the weaker arm and hand could be used strategically as an assist to the stronger arm and hand or even as an assist when using assistive devices such as those designed to augment communication or as walking aids. Sometimes even small changes in a child's ability or even willingness to use a weaker (less-functional) arm and hand can open new opportunities for overall participation. For example, one child treated learned to control a power wheel chair with the weaker arm and hand which allowed his more functional arm and hand to be available for other important activities, such as holding a cup when moving from the kitchen to the table.

It is also striking that many of the lowest functioning children gained the highest number of new behaviors on the EBS. In fact, the overall number of new behaviors gained across this clinical cohort was highly similar to the reported changes on the EBS in previous RCTs $[1,15,16]$ averaging between 10 and 12 new behaviors. In the clinical cohort, MACS level was not a predictor of response because all MACS levels changed at or near this range, but when examined by functional classifications, the children at MACS levels four and five gained just as many and sometimes more than did children at MACS levels II and III. This suggests that children at lower levels can gain many additional behaviors, even when we know they face greater challenges at the start of treatment. This becomes more poignant when you consider that, historically, much of the research about CIMT for both adults and children has included only individuals with preset level abilities placing them into the more moderate (rather than severe) levels of functional classifications.

\subsection{Limitations}

The protocol completed with these families is one of the most intensive protocols available, and we readily recognize that in the confines of the current rehabilitation community most clinicians cannot realistically re-assign their time to accommodate the high number of treatment hours for ACQUIREc. In addition, there are still many unanswered questions about the optimal dosage or intensity of CIMT, with some trials reporting efficacy with lower dosages. Understanding dosage is perhaps the biggest challenge facing CIMT researchers and clinicians, but we must also recognize what we can learn from these higher dosage levels as we disseminate CIMT into new areas. For example, this report included treatment outcomes for children with quadriparesis. The higher dosage model may be useful for children with quadriparesis because they start at a lower functional level and may need more therapy with feedback and opportunities for massed practice of new skills; the higher dosage may be critical to provide the same possibilities for change. This cohort clinical study indicates these children can tolerate and benefit from high therapy dosages.

Another limitation of this study is that the sample of children are from families who sought out this level of intensive services and therefore had the resources to manage a 1-month intensive treatment process. A treatment at this intensity requires significant amounts 
of resources and attention from therapists, the treated child, and from family members who must make life accommodations for it to occur. We do not know how generalizable these findings might be to other types of families in community clinical settings. Finally, we acknowledge that our clinics represent university-based endeavors, with a high commitment to knowledge generation; further, our clinics had the expertise on site from highly experienced therapists. This may have served to enhance the fidelity of CIMT implementation and perhaps to drive up the positive outcomes.

\section{Conclusion}

The dissemination and translation of pediatric CIMT into clinical practice for a broad group of children with hemiparesis and asymmetric upper extremity function is at a crossroads. There are many protocol versions of CIMT. We think it is incumbent on those implementing these protocols to maximize our continued learning from implementing CIMT in diverse clinical and community settings. Gathering Practice-Based Evidence about the impact of various CIMT protocols has the potential to inform expansion of these evidence-based practices. These findings enhance the understanding that pediatric CIMT can be applied to children with both hemiparesis and quadriparesis as part of specialty clinics and lead to significant functional benefits.

\section{Conflict of interest}

The authors have no conflict of interest to report.

\section{References}

[1] DeLuca SC, Case-Smith J, Stevenson R, Ramey SL. Constraint-induced movement therapy (CIMT) for young children with cerebral palsy: Effects of therapeutic dosage. $J$ of Ped Rehab Med. 2012: 5: 133-142. DOI: 10.3233/PRM2012-0206.

[2] Ramey SL, DeLuca SC. History and definition of Pediatric Constraint-Induced Therapy (P-CIMT). In Handbook of Pediatric Constraint-Induced Movement Therapy (CIMT): A Guide for Occupational Therapy and Health Care Clinicians, Researchers, and Educators. 2013. The American Occupational Therapy Association. Bethesda MD.

[3] Ramey SL, Coker-Bolt P, DeLuca SC. Operationalizing pediatric constraint-induced movement therapy (P-CIMT): The core principles to inform training P-CIMT therapists, measuring fidelity of implementation, and developing post-treatment plans. In Handbook of Pediatric Constraint-Induced Movement Therapy (CIMT): A Guide for Occupational Ther- apy and Health Care Clinicians, Researchers, and Educators. 2013. The American Occupational Therapy Association. Bethesda MD.

[4] DeLuca SC, Ramey SL, Trucks MR, Lutenbacher R, Ainsworth D. The ACQUIREc protocol: What we have learned from a decade of delivering a 'signature' form of pediatric constraint-induced movement therapy (P-CIMT). In Handbook of Pediatric Constraint-Induced Movement Therapy (CIMT): A Guide for Occupational Therapy and Health Care Clinicians, Researchers, and Educators. 2013. The American Occupational Therapy Association. Bethesda MD.

[5] Novak I, Mcintyre S, Morgan C, Campbell L, Dark L, Morton N, Stumbles E, Wilson SA, Goldsmith S. Systematic review of interventions for children with cerebral palsy: State of the evidence. Dev Med \& Child Neurol. 2013: 55: 885-910. DOI: 10.111/dmcn. 12244

[6] Novak I. Evidence-Based diagnosis, health care, and rehabilitation for children with cerebral palsy. $J$ of Child Neurology. 2014: 29(8): 1141-1156. DOI: 10.1177/0883073814535503.

[7] Hoare B, Imms C, Carey L. Constraint-induced movement therapy in the treatment of upper limb in treatment of children with hemiplegic Cerebral Palsy: A cochrane systematic review. Clin Rehab. 2007: 21: 675-685. DOI: 10.1177/ 0269215507080783

[8] Boyd RN, Morris ME, Graham HK. Management of upper limb dysfunction in children with cerebral palsy: A systematic review. Europ J of Neur. 2001: 8(S5): 150-166. DOI: 10.1046/j.1468-1331.2001.00048.x.

[9] Taub E, Uswatte G, Pidikiti R. Constraint-induced movement therapy: A new family of techniques with broad application to physical rehabilitation - a clinical review. J of Rehab Research \&Dev. 1999: 36(3): 237-251.

[10] Reiss AP, Wolf SL, Hammel EA, McLeod EL, Williams EA. Constraint-induced movement therapy (CIMT): Current perspectives and future directions. Stroke Research \& Treatment. 2012: 2012: 1-8. DOI: 10.1155/2012/159391.

[11] Ramey SL, Coker-Bolt P, DeLuca SC. Handbook of Pediatric Constraint-Induced Movement Therapy (CIMT): A Guide for Occupational Therapy and Health Care Clinicians, Researchers, and Educators. 2013. The American Occupational Therapy Association. Bethesda MD.

[12] Eliasson AC, Krumlinde-Sundholm L, Gordpn AM, Feys H, Klingels K, Aarts PB, et al. Guidelines for future research in constraint-induced movement therapy for children with unilateral cerebral palsy: An expert consensus. Dev Med Child Neurol. 2014: 56(2): 125-137. DOI: 10.1111/dmcn.12273V.

[13] Horn SD, Gassaway J. Practice-based evidence study design for comparative effectiveness research. Medical Care. 2007: 45(10, 2): S50-58.

[14] Ammerman A, Smith TW, Calancie L. Practice-based evidence in public health: Improving, reach, relevance, and results. Annu Rev Public Health. 2014, 35, 47-63.

[15] DeLuca SC, Echols K, Ramey SL. ACQUIREc Therapy: A Training Manual for Effective Application of Pediatric Constraint-Induced Movement Therapy. 2007. MindNurture. Hillsborough NC.

[16] DeLuca SC, Echols K, Law C, Ramey S. Intensive pediatric constraint-induced therapy for children with cerebral palsy: A randomized controlled crossover trial. J of Child Neuro. 2006: 1: 931-938. PMID: 17092457.

[17] Taub E, Ramey SL, DeLuca SC, Echols K. Efficacy of constraint-induced (CI) movement therapy for children with cerebral palsy. Pediatrics. 2004: 113(2): 305-312. PMID: 14754942 . 
[18] Case-Smith J, DeLuca SC, Stevenson R, Ramey SL. A multi-center randomized clinical trial of pediatric constraintinduced therapy in children with cerebral palsy. $J$ of Occup Therapy. 2012: 66(1): 15-23. PMID: 22389937.

[19] Wallen M, Bundy A, Pont K, Ziviani J. Psychometric properties of the Pediatric Motor Activity Log used for children with cerebral palsy. Dev Med Child Neurol. 2008: 51: 200208. DOI: 10.1111/j.1469-8749.2008.03157x.

[20] Holmefur MM, Krumlinde-Sundholm L. Psychometric properties of a revised version of the Assisting Hand Assessment (Kids-AHA 5.0). Dev Med Child Neurol. 2016: 58: 618-624.

[21] Eliasson A, Krumlinde-Sundholm L, Rosbald B, Beckung E, Arner M, Ohrvall A, Rosenbaum R. The Manual Ability Classification System (MACS) for children with cerebral palsy: Scale development and evidence of validity and reliability. Dev Med Child Neurol. 2006: 48: 549-554.
[22] Palisano R, Rosenbaum P, Walter S, Russel D, Wood E, Galappi B. Development of and reliability of a system to classify gross motor function in children with cerebral palsy. Dev Med Child Neurol. 1997: 39: 214-223.

[23] Sakzewski L, Ziviani J, Abbott D, MacDonnell RA, Jackson GD, Boyd R. Randomized trial of constraint-induced training on activity outcomes of children with congenital hemiplegia. Dev Med Child Neurol. 2011: 53: 313-320.

[24] Islam M, Nordstrand L, Holmstrom L, Kits A, Forssberg H, Eliasson A. Is outcome of constraint-induced movement therapy in unilateral cerebral palsy dependent on corticomotor projection pattern and brain lesion characteristics. Dev Med Child Neurol. 2013: 56: 252-258. 